\title{
Embodied Listening Modes as Part of Habitual Music Media Orientations: Relating Young Adults' Audio Technology Use with Their Music Socialization and Taste Preferences
}

\author{
STEFFEN LEPA, Technische Universität Berlin, Germany \\ MARKUS SEIFERT, Universität Erfurt, Germany
}

\begin{abstract}
Music listening in everyday life is nowadays typically characterized by trans-media use: Most young adults in Germany use several different technologies for accessing, storing, sharing, and listening to their music. Nevertheless, there exist two relatively homogenous large musicrelated media user groups within the younger birth-cohorts: The 'Digital Mobilists' exhibit a rather narrow 'audio repertoire', concentrating on radio, notebook, mobile devices, internal speakers and headphones. Conversely, the 'Versatile Audiophiles' have a broader audio repertoire encompassing also $\mathrm{HiFi}$ stereo units, various storage media, HiFi headphones and separate loudspeakers of various kinds. The paper is describing a mixed-methods study which tries to explain young adults' membership in one of these two 'communicative figurations' both by a) logistic regression on socio-demographics, media socialization and musical taste preferences employing a first-year student sample as well as by b) analyzing six biographicepisodic interviews conducted with adolescents and emerging adults of both media user types in focus. On the one hand, our results show the ways in which the two identified everyday music listening modes are grounded in highly-ritualized, embodied situated practices with audio media technologies which are felt to enrich otherwise rather 'non-musical' social activities. On the other hand, the results demonstrate the importance of gender and social milieu, early music listening experiences with audio media and the apparently body-related implications of certain music genres for explaining and understanding habitual audio media use in later adult life.
\end{abstract}

\section{KEYWORDS}

Media Socialization; Media Practice; Mediatization; Mixed-Methods; Aesthetic Schemes 


\section{INTRODUCTION}

\subsection{Desiderates of Audio Repertoire Research Regarding Early Adults}

Everyday music listening is nowadays typically characterized by trans-media use: According to the overall findings of a pilot study conducted by the first author (Lepa, Hoklas and Weinzierl 2014) ${ }^{1}$, most Germans use several different technologies for accessing, storing, sharing, and listening to their personal music. According to the population-representative study conducted in the beginning of 2013, there exist two relatively homogenous large musicrelated media user groups within the younger birth-cohorts of adolescents and young adults (born approximately after 1980): The Digital Mobilists exhibit a rather narrow audio repertoire, concentrating on radio, notebook, mobile devices, internal speakers and headphones. Conversely, the Versatile Audiophiles have a broader audio repertoire encompassing also $\mathrm{HiFi}$ stereo units, various types of storage media, HiFi headphones and separate loudspeakers of various kinds. As results of a regression analysis demonstrated, apart from minor birth cohort differences, female gender and a lower household income are mainly able to explain membership in the Digital Mobilist type. While the latter finding delivers a rather straightforward explanation, the actual social mechanism that leads to 'being female' also predicting a narrower and rather 'mobile' repertoire for young music consumers remains unclear, especially since a comparable 'gender split' was not observed within any of the earlier birth cohorts of middle and late adults (Lepa, Hoklas and Weinzierl 2014). Furthermore, a larger portion of variance in the present audio repertoire composition of the late adolescent and early adult German population still remains empirically unexplained. Since the Latent Class Analysis (LCA) approach employed in the SMuM study for audio repertoire classification allows transferring the estimated classification rules to new datasets (Collins and Lanza 2010), we performed a follow-up study that aims at better explanation and understanding of emerging adults' audio usage patterns.

\subsection{State of Research Concerning Audio Repertoire Genesis}

Adopting the theory of generations as carriers of social change by Karl Mannheim (1952), we postulated that alongside the mediatization of everyday music listening ${ }^{2}$, larger and partly opposing societal habitus formations are formed that manifest themselves empirically in the form of similar prototypical audio repertoires observable on societal level (Lepa, Hoklas and Weinzierl 2014): Following Mannheim's line of thinking, the human 'carriers' of these communicative figurations $^{3}$ (Hepp 2013) are thought to have developed different and partly even opposing 'practical answers' to the material-symbolic action spaces of music listening they experienced in their formative years of childhood and youth. The resulting music media orientations are composed of collectively shared implicit habits, experiences, action scripts, schemes, expectations, imaginations and feelings towards what is (situationally) expected

\footnotetext{
${ }^{1}$ The project 'Music and Media Survey' (in German: 'Survey Musik und Medien. Empirische Basisdaten und theoretische Modellierung der Mediatisierung alläglichen Musikhörens in Deutschland') was funded by the German Research Foundation (DFG) with reference number LE 3096/1-1 as part of the national priority research program 1505 'Mediatized Worlds'.

${ }^{2}$ The concept of 'mediatization' refers to an assumed societal meta-process through which different social worlds are more and more influenced by ever-new media technologies in the course of human history, resulting in long-term social change alongside technological change in these worlds (Krotz 2014).

${ }^{3}$ According to Hepp $(2013,623)$, 'communicative figurations' are a special form of social formations that may historically emerge 'as patterns of processes of communicative interweaving that exist across various media and have a "thematic framing" that orients communicative action.' In the present case, the thematic framing is people's desire to listen to music in their everyday life.
} 
from a musical experience with media and how it is to be realized. As our pilot study demonstrated, they form a latent generic 'audio media grammar' that is upheld by with certain inertia through lifetime, not totally precluding slow adaptations of the individual audio repertoires due to technically or socially initiated changes in personally accessible media environments (Lepa, Hoklas and Weinzierl 2014).

First aim of the present exploratory mixed-method study is to extend this state of research by examining the specific influence of two different types of socialization experiences on the formation of music media orientations leading to similar audio repertoires: In line with Krämer (2013), we assume music media orientations to be acquired alongside the general socialization into media music culture, typically starting in childhood, when first experiences with media are made together with the parents as primary socialization agents (Baacke, Sander and Vollbrecht 1990). This on the one hand may occur in informal audio socialization contexts like initial media-based listening to music and audio plays with parental allowance and support (Dollase 1998). But it may likewise happen in the context of more formalized music socialization contexts (Lenz 2013) when audio media are employed in music education in school or alongside learning a musical instrument. With increasing age and transition to puberty, influence of parents and school should then diminish in favor of the peer-group as secondary socialization agent, typically accompanied by an increasing degree of selfmonitoring and self-control of the agent in audio media selection and use in terms of time, situation, content and technology (Müller-Bachmann 2002). Additional to findings that musical educational practices in family and school are partly gendered (Hallam 2002), there are also hints to gender-specific socialization experiences with audio media in family and peer-group (Keightley 2003). Therefore, by estimating the specific role of different types of socialization within our explanatory model, the gendering of audio repertoire composition may possibly be understood better, too.

\subsection{Research on Socialization of Musical Taste and its Relation to Media Use}

A further line of theoretical reasoning to be explored in the current paper considers the idea that musical taste preferences might be a product of the very same music media orientations formed in childhood and adolescence. While from an everyday-life understanding, 'musical taste' is often interpreted as individual liking or avoidance of certain titles, artists or whole musical genres, cultural sociologists like Schulze (1992) have convincingly argued that musical taste preferences given by musical genre labels might in fact represent higher-order and collectively shared everyday aesthetic schemes that are not only directed on intrinsic aspects of music (genre-typical musical style, affective expression and impact, musical virtuosity), but also on extra-musical multi-sensual and social aspects of musical genres such as typical receptive modes, accompanying social scripts, related fashion and dance styles, associated social classes, generations, ethnics and milieux, that may altogether serve for cultural distinction (Lepa and Seifert, in press). Such schematic generalizations that orient aesthetic experiences with certain types of music may comprise style-related cognitive modes of analyzing, interpreting and aesthetically enjoying music, the evocation of memories from earlier times of exposure, the promotion of associations with certain artists, genres and cultural scenes, but also physiological effects of specific styles and various accompanying scripts of social action during listening (Schäfer and Sedlmeier 2010; Schramm 2005). Therefore, it seems not far-fetched to assume that habitual reliance on certain audio media devices may also form part of them. 
Vice-versa, research in music socialization localizes the origins of adults' musical taste preferences (apart from individual biologic-genetic dispositions and related bodily needs) in very similar psycho-social mechanisms to those assumed to generate later music media orientations: Development of basic taste preferences and faculties of discrimination (Miranda et al. 2015) is also said to happen in informal audio socialization contexts like listening to the radio, the HiFi stereo, or via watching music shows in television, typically under sanctioning of the parents (Kunz 1998), but also alongside formalized music education in family (Gebesmair 2001) and school (McPherson 2009), which form the primary socialisation agents within the first life decade. Their influence then gradually diminishes with puberty, and is increasingly substituted by the peer-group as secondary socialization agent (Jost 1982). The resulting preference schemes are assumed to be gender and milieu-specific (Gebesmair 2001) and to reach quasi-stability and growing inertia with the end of the second live decade, resulting in a crystallized musical taste scheme representing an elaborated musical identity of the grown-up (Reinhardt and Rötter 2013). In spite of the obvious familiarities between both theoretical concepts in focus, there is yet just one empirical study concerning the kind and intensity of their possible relationships which employs survey data from Chile (Leguina, Arancibia-Carvajal and Widdop 2015). While its results deliver first empirical evidence about existing correlations between musical taste and audio technology use in the general population, it does not systematically control for cohort influences and is therefore not able to differentiate generational influences from socialization-related heterogeneities. Hence, we analyze the latter in the present paper with a mixed-methods study focussing on adolescents only.

\subsection{Summary of Research Questions and Employed Mixed-Methods Logic}

In sum, with regards to early adults audio repertoires we hypothesize (H1) that apart from already identified socio-demographic determinants, the intensity of music and audio media related socialization experiences explains additional variance in audio repertoire composition of young adults and might also explain parts of the found gender influences on their audio repertoire composition. Taking this assumption into account, we additionally hypothesize $(\mathrm{H} 2)$ the existence of significant correlations between audio repertoire class membership and individual musical taste preferences since both can be assumed to be influenced by the same underlying music media orientations. In order to test both assumptions, we firstly conduct a quantitative survey study with a student sample that initially identifies audio repertoire class membership ${ }^{4}$ for each participant and then estimate a stepwise logistic regression model that operationalizes our hypotheses. Due to our survey sample being predominantly composed of individuals from a higher-educated background, we unfortunately cannot test for heterogeneities related to socio-economic background or educational milieu in this part of the study, though we would expect such heterogeneities to occur based on the state of literature depicted above. In a qualitative follow-up study, we then analyse six interviews with late adolescents and young adults of both audio repertoire classes in order to enrich the interpretations of the empirical relations found by the survey study. Hereby, we take into account that the concrete audio technologies applied and the musical content mobilized in the everyday as 'technologies of the self' may partly adhere to strongly situation-specific logics (DeNora 1999) and often also include practices that very much draw on the body (Quiñones 2013). Therefore, situated contiguities between musical genres and audio technologies, as

\footnotetext{
${ }^{4}$ In the framework of Latent Class Analysis (LCA), the term 'class' is a technical term denoting a subgroup of individuals with relative similar data patterns, so its meaning is comparable (though not identical) to the terms 'cluster' or 'type'. To prevent any confusion with the notion of social class in socio-economic and cultural analyses, we will always speak of audio repertoire classes throughout this paper.
} 
well as the role of the body are put into special empirical focus in the spirit of newer discussions in media dispositif research (Lepa, Krotz and Hokla 2014).

\section{METHODS}

\subsection{Survey Study}

\subsubsection{Sampling Procedure}

The quantitative part of the study was conducted in form of a paper-and-pencil questionnaire as part of introductory lectures in early 2014 at the universities of Weimar and Erfurt, with the obtained sample comprising $\mathrm{n}=699$ first-year bachelor and master students of various study subjects from nearly all faculties of both universities.

\subsubsection{Questionnaire Structure}

The first part of the questionnaire asked for audio technologies used habitually in 2013. This was done analogous to Lepa, Hoklas et al. (2014), but with only the 13 most differentiating of the original 44 Items of the SMuM study in order to shorten the overall procedure. Accordingly, participants where asked on five-point ordinal scales ('never', 'once a year', 'monthly', 'weekly', 'daily') with which intensity they had employed CDs, records, cassettes, MiniDiscs, radio, memory sticks / hard drives, music streaming providers, music cloud, music recommendation services, video hosting services, websites or social networks for listening to music during the course of the prior year. A further part operationalized the intensity of music and media related socialization practices in childhood and youth. Therefore, participants had to fill five Likert items (see figure 1) that either represented the intensity of formalized music education (music socialization) or the intensity of informal socialization practices with audio media (audio socialization). Participants were able to gradate their answer on a five point scale ranging from 'never' to 'very often', apart from two items that were asked in dichotomous mode due to logical reasons ('learned an instrument', 'regularly sang in a choir'). The third part of the questionnaire asked for participants' musical preferences by asking for the individually felt affinity to 15 presented musical major genres (see table 3), drawing on a similar catalogue from Taubert (2006). Participants were again able to gradate their liking on a five-point-scale ranging from 'very much dislike' to 'very much like'.

\subsubsection{Statistical Analyses}

The first part of statistical analysis was to classify the study participants into the audio repertoire classes identified in the pilot study on basis of the given data on their habitual audio media use. Thereto, the item-specific conditional probability thresholds for audio repertoire class memberships gathered in the population representative SMuM-study (Lepa, Hoklas and Weinzierl 2014) were combined with the present data by employing the Bayes-Theorem to infer the survey participants' most probable audio repertoire class membership on basis of their individual data patterns, following the procedures explained in Collins \& Lanza (2010). Afterwards, and with regards to the given research questions, only members of audio repertoire classes $\mathrm{C} 1$ and $\mathrm{C} 2$ were selected for a subsequently conducted stepwise logistic regression model that aimed at explaining membership in one of the two classes by sociodemographics (step 1), intensity of music and audio socialization (step 2) and musical taste 
preferences (step 3). The construct variables introduced in step 2 and step 3 were previously corrected for measurement error by conducting confirmatory and EFA/ESEM factor analysis (Marsh et al. 2009), respectively. ESEM is a relatively new approach that allows the direct implementation of EFA-factors (exploratory factors containing multiple cross-loadings) in structural equation models for means of hypothesis testing. All statistical analyses were realized employing the MPlus 6.12 structural equation modelling software package (Muthén and Muthén 2010), with all questionnaire items interpreted as ordinal, resulting in employment of the robust WLSMV algorithm for all model parameter estimations and the use of weighted least squares as the factor extraction method in the EFA/ESEM factor analysis.

\subsection{Interview Study}

\subsubsection{Selection of Informants}

The second part of the study was a narrative interview analysis. The six informants selected formed part of a larger sample that had been contacted and interviewed when conducting the nationwide SMuM study (Lepa, Hoklas and Weinzierl 2014) on basis of their very 'classtypical' personal audio repertoire profiles. To complement results of the present survey study, we generated a subsample drawing only on informants from the two 'youngest' audio repertoire classes. Three of them had been identified as typical Digital Mobilists and the other three as Versatile Audiophiles before. To the time of the interview (late summer 2013) their age ranged between 15 and 28 (see table 1). The gender composition of the sample approximately reflects the true population proportions in the high-education segment of that age. The interviews were conducted in the informants' rooms within their families' flats and houses, took about 1-2 hours and every participant received a monetary compensation. ${ }^{5}$

Table 1: Composition of the Interview Study Sample

\begin{tabular}{|l|l|l|}
\hline Audio Repertoire Class & C1 - Versatile Audiophiles & C2 - Digital Mobilists \\
\hline \multirow{3}{*}{ Informants' Data } & Tim, 28, male, student & Tilla, 26, female, student \\
& Noah, 17, male, college & Katharina, 18, female, apprentice \\
& Malek, 15, male, pupil & Kareem, 17, male, pupil \\
\hline
\end{tabular}

\subsubsection{Interview Guide Structure}

Interviews took place in the informants' home and started with asking them to delineate their first experiences with audio media in their life course. Thereafter, participants were encouraged to freely narrate their whole personal audio media biography up to the present time and to picture typical nowadays situations of audio media use and to describe the procedures, practices and experiences related to it. Our visit ended with an 'ethnographic walk' through the audio equipment present in the flat or house and the request to demonstrate its practical use and comment and elaborate on it. The interviews were audio recorded with informed consent of the participants.

\footnotetext{
${ }^{5}$ We thank our colleagues Anne-Kathrin Hoklas and Martin Guljamow for conducting the interviews and helping with their interpretation as part of their qualification as doctoral / graduate student in the Audio Communication Group. Transcript quotations in this article were translated to English with the aim of preserving the original colloquial narrative style of the interviews.
} 


\subsubsection{Grounded Theory Analysis}

The obtained audio recordings were transcribed into word processor files with all references to informants' names and locations anonymized. Resulting transcripts were then initially hermeneutically open-coded with the aim of identifying parts that describe either aspects of general music media orientations (S1) or situated music listening dispositifs (S2). Drawing on Grounded Theory, we then developed and validated several key categories within our interpretive research group that describe homologies we found within and across cases on both realms. In a final step, we contrasted the category memberships with the audio repertoire class memberships in order to find out possible regularities.

\section{RESULTS}

\subsection{Survey Study}

\subsubsection{Latent Classification Results}

The proportions of audio repertoire class members found after classification of the present student sample gave an expected result from the perspective of the whole population sample in Lepa, Hoklas, et al. (2014): Most of the study participants belonged to either of the two classes in theoretical focus (see table 2), leading to a subsample for the later logistic regression analysis of $\mathrm{n}=662$ (56.2\% females) with 184 Versatile Audiophiles and 478 Digital Mobilists.

Table 2: Results of Audio Repertoire Classification with the Survey Study Sample

\begin{tabular}{|l|l|l|}
\hline Audio Repertoire Class & Absolute Frequencies & Relative Frequencies \\
\hline C1: Versatile Audiophiles & 184 & $26.3 \%$ \\
\hline C2: Digital Mobilists & 478 & $68.4 \%$ \\
\hline C3: Selective Traditionalists & 29 & $4.1 \%$ \\
\hline C4: Selective Adopters & 6 & $0.9 \%$ \\
\hline C5: Versatile Traditionalists & 0 & $0 \%$ \\
\hline C6: Radio Traditionalists & 1 & $0.1 \%$ \\
\hline Ambiguous classification & 1 & $0.1 \%$ \\
\hline
\end{tabular}

Class memberships inferred by Bayes-Theorem and conditional item-thresholds from Lepa, Hoklas, et al. (2014)

\subsubsection{Results of Factor Analyses}

The items scores gained on the two scales for music and media socialization intensity underwent confirmatory factor analysis to confirm dimensionality structure. Since we assumed a common influence of parents as primary socialization agents with regards to music related socialization, we freed the respective covariances between the two items addressing parental influence (see figure 1), resulting in a very good fit for the overall measurement model $\left(\mathrm{X}^{2}=4.353 ; \mathrm{df}=3 ; \mathrm{p}=0.23\right.$; $\left.\mathrm{RMSEA}=0.025 ; \mathrm{CFI}=0.997 ; \mathrm{WRMR}=0.366\right)$. 


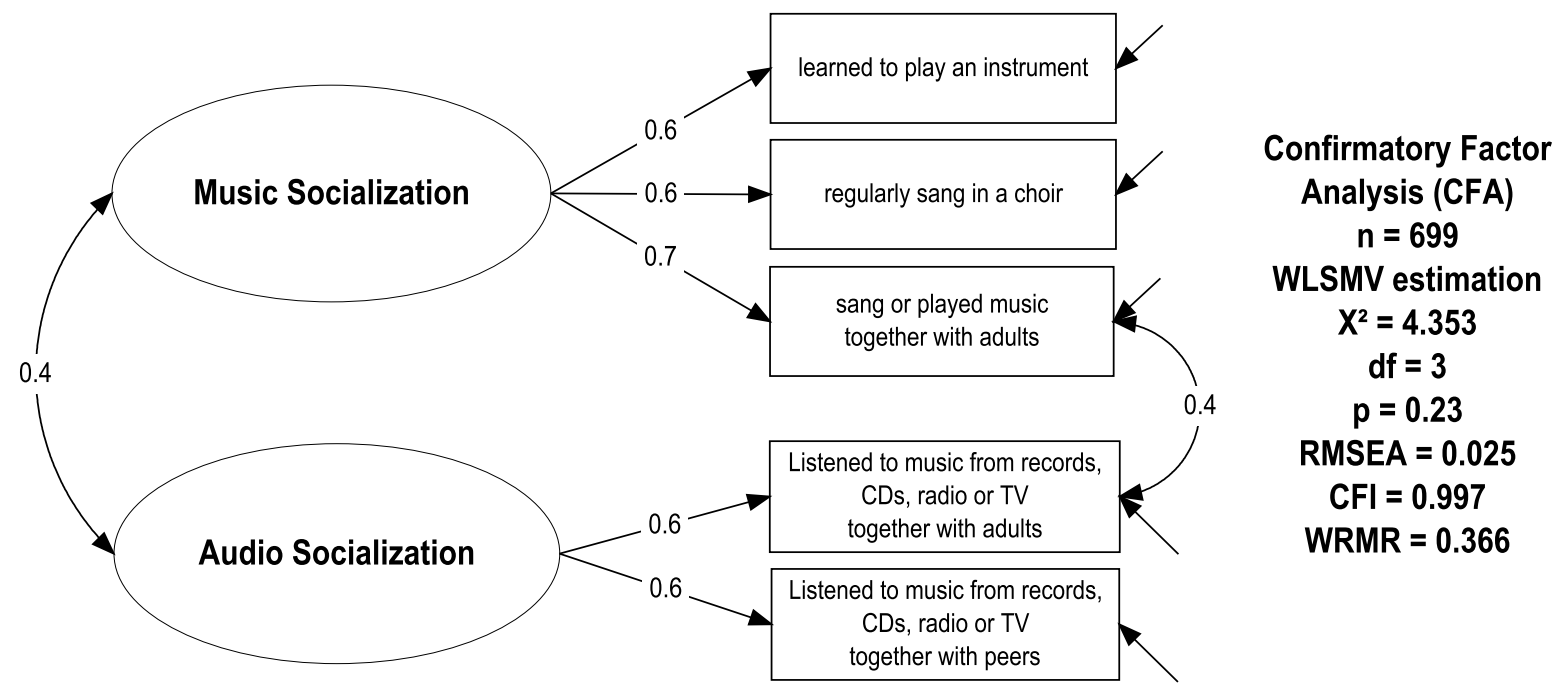

Figure 1: Confirmatory Factor Analysis for Music and Audio Socialization

Due to our theoretical assumptions that conceive of music genre labels as polyvalent and of musical preference dimensions as 'fuzzy meta-genres' representing higher order everyday aesthetic schemes (Lepa and Seifert, in press), individual scores and dimensionality of musical taste preferences were analysed employing exploratory factor analysis (EFA) with oblique Crawford-Ferguson Varimax rotation, thereby allowing multiply item loadings and factor inter-correlations in order to reflect our theoretical assumptions about the construct. Combination of the Scree and Kaiser criterion led us to the decision for a five-factor solution in terms of achieving most parsimonious validity of the measurement model which at the same time exhibited a good fit to the data $\left(\mathrm{X}^{2}=116.867\right.$; df $=40 ; \mathrm{p}<0.001$; RMSEA $=$ 0.052; CFI $=0.979 ;$ WRMR $=0.532$ ). Subsequent inspection of obtained factor loading matrix (see table 3) showed a dimensional structure very similar to the five-factor MUSIC model of musical taste preferences as empirically developed by Rentfrow, Goldberg, \& Levitin (2011). Therefore we adopted their factor interpretation to our results (see Lepa and Seifert, in press, for a more extensive interpretation of the factor structure) and labelled the received musical taste dimensions as Mellow, Unpretentious, Sophisticated, Intense and Rhythmic ${ }^{6}$. Final inspection of factor-intercorrelations resulted in only minor correlations between the different taste dimensions, with $\mathrm{r}=0.26$ between Rhythmic and Mellow as well as $\mathrm{r}=0.17$ between Sophisticated and Intense being the highest obtained scores.

\subsubsection{Inter-Correlation Analysis and Stepwise Logistic Regression Model}

In the final step of analysis, we tried to explain membership probability in one of the two selected audio repertoire classes by a stepwise logistic regression procedure realized by help of structural equation modelling (see table 4 for stepwise beta estimations and overall model fit). Before, we had checked the inter-correlations of all predictor variables for possibly problematic multi-collinearity that could endanger estimation precision of the logistic regression. Highest correlation scores were found with female gender and music socialization $(\mathrm{r}=0.45)$, affinity for Sophisticated music and intensity of music socialization $(\mathrm{r}=0.42)$, as well as with female gender and affinity for Mellow music $(r=0.40)$. Hence we concluded that inter-correlations between the predictor variables were still within an acceptable range.

\footnotetext{
${ }^{6}$ Rentfrow, Goldberg and Levitin (2011) labelled their last obtained musical preference factor 'Contemporary', but from our view, this seems like a mere placeholder for groove-oriented electronic dance music (EDM) genres that from our point of view would better deserve the label 'Rhythmic'.
} 
Table 3: EFA Factor Loading Matrix for Students' Musical Genre Preferences

\begin{tabular}{|l|c|c|c|c|c|}
\hline Loadings (standardized): & Intense & Unpretentious & Sophisticated & Rhythmic & Mellow \\
\hline German-speaking Rock \& Pop & $\mathbf{0 . 7 0}$ & $\mathbf{0 . 1 8}$ & $\mathbf{- 0 . 0 9}$ & -0.03 & 0.03 \\
\hline English-speaking Rock \& Pop & $\mathbf{0 . 8 3}$ & 0.02 & -0.02 & -0.02 & $\mathbf{0 . 0 8}$ \\
\hline Alternative Rock / Indy Music & $\mathbf{0 . 4 4}$ & $\mathbf{- 0 . 3 1}$ & $\mathbf{0 . 4 0}$ & $\mathbf{0 . 1 0}$ & $\mathbf{- 0 . 1 8}$ \\
\hline Chart Hits / German 'Schlager' & $\mathbf{0 . 0 8}$ & $\mathbf{0 . 9 4}$ & $\mathbf{- 0 . 0 8}$ & $\mathbf{0 . 0 4}$ & 0.02 \\
\hline Traditional German / Brass Music & -0.04 & $\mathbf{0 . 6 2}$ & $\mathbf{0 . 4 1}$ & 0.04 & $\mathbf{- 0 . 0 8}$ \\
\hline Country & $\mathbf{0 . 1 2}$ & $\mathbf{0 . 2 5}$ & $\mathbf{0 . 4 3}$ & -0.17 & 0.01 \\
\hline Jazz / Blues & -0.03 & -0.05 & $\mathbf{0 . 7 0}$ & 0.03 & $\mathbf{0 . 1 5}$ \\
\hline World Music / Folk / Chanson & $\mathbf{0 . 1 1}$ & 0.02 & $\mathbf{0 . 6 8}$ & -0.02 & -0.02 \\
\hline Classical Music / Opera & $\mathbf{- 0 . 0 8}$ & 0.07 & $\mathbf{0 . 6 5}$ & -0.02 & 0.03 \\
\hline Spiritual / Gospel / Meditation & 0.04 & $\mathbf{0 . 1 7}$ & $\mathbf{0 . 3 9}$ & -0.07 & $\mathbf{0 . 3 8}$ \\
\hline Techno / House & -0.02 & $\mathbf{0 . 0 5}$ & $\mathbf{- 0 . 0 7}$ & $\mathbf{0 . 8 9}$ & 0.05 \\
\hline Electro-Pop / Electronica & 0.01 & 0.00 & $\mathbf{0 . 0 9}$ & $\mathbf{0 . 8 3}$ & 0.00 \\
\hline Hip Hop / Rap & $\mathbf{- 0 . 0 7}$ & -0.07 & -0.03 & $\mathbf{0 . 2 9}$ & $\mathbf{0 . 4 2}$ \\
\hline Soul / R'n'B / Funk / Black Music & $\mathbf{0 . 0 5}$ & -0.02 & 0.03 & 0.02 & $\mathbf{0 . 9 5}$ \\
\hline Heavy Metal / Hardcore & 0.03 & 0.00 & $\mathbf{0 . 1 9}$ & -0.06 & $\mathbf{- 0 . 3 0}$ \\
\hline Ording EFA, & & & & \\
\hline
\end{tabular}

Ordinal EFA, $n=699$, WLSMV estimation, oblique CF-Varimax rotation, $p<0.05$ significant loadings in bold

Results of the first logistic regression model (step 1) confirm that the scores on the manifest variables birth cohort and gender significantly determine being member of either audio repertoire class, with younger and female participants being more probable to be members of the Digital Mobilists. This explains about $\mathrm{R}^{2}=8 \%$ of audio repertoire class membership probabilities. The step 2 model shows an additional significant influence of the intensity of audio socialization on audio repertoire class membership: Students with more experience of peer and parental-guided introduction to self-selected music listening exhibit a higher probability of being Versatile Audiophiles, while intensified music socialization does not seem to play a substantial role in this regards. Meanwhile, introduction of this new variable block leaves the coefficients of model 1 virtually unaltered and leads to a cumulated overall variance explanation of $\mathrm{R}^{2}=13 \%$.

The step 3 model finally demonstrates additional significant relationships between individual musical taste preferences and being member of one of the two audio repertoire classes in focus: While a preference for Unpretentious and Rhythmic music is slightly more prevalent among the Versatile Audiophiles, a preference for Mellow music is a bit more typical for the Digital Mobilists. At the same time, negative suppressor effects with regards to the variables of block 1 and 2 were observed: The effect of gender found in model 1 falls below statistical significance and the audio socialization effect found in model 2 is substantially reduced in magnitude, leading to the conclusion that these both may be partly spurious causal relationships that can be also explained by correlations of the same variables with the dimensions of musical preferences. Altogether, the final model (see table 4) is able to explain $\mathrm{R}^{2}=21 \%$ in audio repertoire class membership probabilities. 
Table 4: Stepwise Logistic Regression for Explaining Audio Repertoire Class Membership

\begin{tabular}{|c|c|c|c|}
\hline & $\begin{array}{c}\text { Model 1: } \\
\text { socio-demographics }\end{array}$ & $\begin{array}{c}\text { Model 2: } \\
\text { socio-demographics } \\
\text { and socialization }\end{array}$ & $\begin{array}{c}\text { Model 3: } \\
\text { socio-demographics, } \\
\text { socialization, and } \\
\text { musical preferences } \\
\end{array}$ \\
\hline $\mathbf{X}^{2}$ & 0 & 20.859 & 385.232 \\
\hline df & 0 & 12 & 147 \\
\hline $\mathbf{p}$ & $<0.001$ & 0.053 & $<0.001$ \\
\hline RMSEA & $<0.001$ & 0.032 & 0.045 \\
\hline CFI & 1.000 & 0.985 & 0.952 \\
\hline WRMR & $<0.001$ & 0.560 & 0.831 \\
\hline Predictors: & $\begin{array}{l}\text { Logistic Regression } \\
\text { P (Digital Mobilist } \\
\text { vs. Versatile Audiophile) } \\
\end{array}$ & $\begin{array}{c}\text { Logistic Regression } \\
\text { P (Digital Mobilist } \\
\text { vs. Versatile Audiophile) }\end{array}$ & $\begin{array}{l}\text { Logistic Regression } \\
\text { P (Digital Mobilist } \\
\text { vs. Versatile Audiophile) } \\
\end{array}$ \\
\hline $\begin{array}{l}\text { Birth Cohort } \\
\text { Gender (f) }\end{array}$ & $\begin{array}{l}0.11 \\
0.23\end{array}$ & $\begin{array}{l}0.12 \\
0.25\end{array}$ & $\begin{array}{l}\mathbf{0 . 1 0} \\
0.12\end{array}$ \\
\hline $\begin{array}{l}\text { Music } \\
\text { Socialization }\end{array}$ & & 0.04 & 0.08 \\
\hline $\begin{array}{l}\text { Audio } \\
\text { Socialization }\end{array}$ & & -0.24 & -0.20 \\
\hline Intense & & & -0.06 \\
\hline Unpretentious & & & -0.18 \\
\hline Sophisticated & & & -0.08 \\
\hline Rhythmic & & & -0.15 \\
\hline Mellow & & & 0.21 \\
\hline $\mathbf{R}^{2}$ & 0.08 & 0.13 & 0.21 \\
\hline$\Delta \mathbf{R}^{2}$ & & 0.05 & 0.08 \\
\hline
\end{tabular}

\subsection{Interview Study}

\subsubsection{Embodied Listening Modes and their Biographical Genesis: Key Categories}

After open-coding all narrated descriptions of different modes of accessing, storing and assembling music depicted in the six selected interview transcripts and then contrasting members of both classes, we found two different and clearly discernible principles on which our informants seemed to base their selections and assemblages of the 'right music and audio device' for a given situation. The implementation of these behavioural patterns always embedded music listening within other social activities and was depicted as being regularly related with mood-changes, experience of certain emotions or different types of bodily (re-) actions. Accordingly, we interpret the two identified key categories as embodied listening modes forming the basis of music media orientations that implicitly guide our informants' ways of everyday music listening. While some informants showed knowledge and experience with both modes, we nevertheless found a hierarchical order of importance with every informant. Furthermore, we discovered that habitual dominance of either mode appears to be related to similar formative experiences with music and audio media during our informants' childhood and early youth. 


\section{A: Functional orientation - bodily impact and contextual style (Versatile Audiophiles)}

Informants of the Versatile Audiophile cluster (Tim, Noah, Malek) generally exhibit a very functionalist outlook towards music and audio technologies. From their childhood on, they have shown a keen interest in HiFi stereo technology and the world of music, which they explored together with parents and (predominantly male) friends alongside the ongoing mediatization of the audio world and often also via visiting live concerts. They feel like researchers in the field of music, aiming at creating a perfect aesthetic experience that also encompasses the body through means of audio technology, often through volume and bass. They are always interested in new developments in audio devices and musical style and early learned how to utilize this special knowledge for socializing. Hence, they have served until today as service providers for their peers and parents as 'CD burning master', DJs on parties or playlist compilers. To them, the 'right music and audio device' is a matter of fit to the situation in terms of the 'correct style', even if it may not be a totally adequate expression of their personal situational needs in terms of emotion or personal liking. Accordingly, they will often compile playlists for others and choose playback technology tailored to the overall style of a social situation. For their solipsistic listening, they tend to compile music that will fit to their current phase of interest and own cultural taste, while still being omnivorously (Peterson and Kern 1996) interested. If possible, they will always employ high-quality devices that enable them access to a rich world of music and that afford high quality in times of frequency range, volume and bass. Furthermore, due to their extended embodied experiences with different music styles and audio hardware, they cultivate extensive subjective theories about the special situated affordances of loudspeakers vs. headphones, sound quality of different audio devices and matters of musical style.

\section{B: Expressive orientation - biography, identity and belonging (Digital Mobilists)}

The members of the Digital Mobilist cluster that we interviewed (Tilla, Katharina, Kareem) tend to see music and audio technologies predominantly as means for personal affectivesymbolic expression. In the course of their childhood and in their later adolescent lives, they had very vivid experiences with emotional collective situations that seemed to exhibit a perfect fit to the accompanying music. Hence, they are now continuously trying to symbolically re-create and elaborate the memories and feelings of social belonging and togetherness that were (and still are) formed in the namely situations through the power of music. While this makes them enthusiasts for special songs, artists or types of music, to which they often feel a very intimate relation, this is not accompanied by a special occupation with audio media technologies. They regard them 'only as a means', and typically learned to handle them through older siblings and parents only but not in the peer-group. Accordingly, they even nowadays often draw on the help of 'experts' similar to members of the Versatile Audiophile cluster in order to support them with audio technology or music content they are looking for. With regards to audio technologies, they generally tend to care less about functionality in technical terms or matters of actual bodily effects but focus more on the symbolical value of certain devices and storage media which are related to their personal biographies or those of their significant others. With regards to musical taste, they show a similar behaviour: Genre is seen as a collection of symbolical values. Therefore, expressively oriented listeners may also appear as 'omnivores' (though of a different style) considering the fact that they often not only develop a certain biography-based personal taste but additionally also 'social taste provinces' that they only share with certain long-term significant others like parents or 'best friends' and that may not follow at all their overall aesthetic alignment of their felt musical identity. 


\subsubsection{Situated Music Media Dispositifs: Key Categories}

After focussing on our informants' basic listening orientations we were also able to identify several commonalities within the structure of situated experiences and practices with music across and within cases. The resulting 'music media dispositif structures' (Lepa, Krotz and Hoklas 2014) describe prototypical couplings of social situations, musical styles, specific media devices, accompanying actions and social regimes that address predominantly either of the two embodied listening modes depicted above. Following our research questions in a triangulatory fashion, we continue by giving a concise overview of the content and structure of the four most contrastive music listening dispositif structures we found in terms of musical styles and audio technologies employed.

\section{A: Dancing, Singing and Dreaming when Alone with Unpretentious and Mellow Music}

A practice commonplace to several of our informants from the Digital Mobilist cluster is to listen, sing, elaborate and dream to current favourite hits from the Unpretentious and Mellow taste dimensions while being alone in their room. For realizing this activity at home, typically, a laptop with built-in speakers is employed. Our informant Katharina depicts this way of action as follows:

I: When you say the music is 'in the foreground', what does that mean exactly?

$R$ : Well, that I concentrate very much on it, listen attentively to the lyrics, making my elaborations on it, hum, I dunno, I just imagine some things then. You know, I am kind of a daydreamer, I have to admit. Well, yes, I dunno, sometimes I also sing along or dance through the room, you know, just these kind of things you do when listening to music. Yes.

Similar experiences are described by all interviewed members of the Digital Mobilist cluster, with some also employing their smartphone with built-in speakers as the playback appliance. In all these situations, the music is played back on a rather low or medium volume level. Furthermore, the music genre listened to in these situations varies between chart hits and more mellow and soulful styles. Access to music is realized by tuning into the favourite radio station, subscriptions to style-oriented playlists or by specially tailored playlists composed by downloads through YouTube Converter or music blogs. These lists with a smaller selection of 'current greatest hits' are typically assembled weekly or monthly and often played back with the 'shuffle' function or in a 'skipping through' fashion. Kareem narrates:

R: Well, you know, I got this YouTube channel, and there I have subscriptions with my favorite rappers and singers, then I always watch, hum, for example, whether they published a new album or single. Then I just google it, the new single, adding 'download', because there a certain websites where they provide these new singles or sometimes I find mixtapes including the newest rap from the USA and that's where I then get my music.

The embodied aspects of the music listening related activities in solipsistic situations comprise singing, thinking about the lyrics, making small dancing movements and triggering activation of inner fantasies. Meanwhile, the music is played on a rather lower volume, fitting to the needs of the situation and also enabling singing along in a soft voice. Conversely, members of the Versatile Audiophiles cluster seem to adhere to these types of activities to a much lesser degree, since none of the informants from this cluster described this type of practice. 


\section{B: Soulful Cosiness in the Home with Sophisticated and Mellow Music}

A more seldom, but still important embodied activity of some of Digital Mobilist informants is a kind of ritually enacted cosiness in which single songs or certain albums that have a special biographical meaning are employed in a highly ritualized form to remember and restore certain modes of being-in-the-world and inter-subjectivity. Often these are songs that are related to a certain life period, a special concert event attended or the relationship to a certain person. The chosen musical works stemming from the Sophisticated or Mellow genre are typically listened to repeatedly, often accompanied by a specific liturgy encompassing special light and incense, listening position and locations. As part of these activities, also material storage media like vinyl records, CD albums and cassettes may play a major part, such as for Tilla:

R: Or sometimes, I use to think, oh, a bit of scraping from a vinyl would be nice right now, especially regarding the atmosphere. Hum, in particular when I am thinking, oh, now I wanna have it nice and cosy, but I still gotta do some stuff, but I would really like to take care of it in a nice atmosphere. Then I will in any case turn on a record and candles and... I dunno, somehow do something like that, yes.

While all members of the Digital Mobilists' cluster narrate about this type of situated practice, it seems to be of lesser importance for members of the Versatile Audiophiles. While the latter have certain favourite tunes, too, they nevertheless would only very seldom initiate this type of solipsistic expressive worshipping of certain single artists or songs (in a fan-like fashion), even less by using (from their point of view) 'old fashioned' media. Nonetheless, Versatile Audiophiles would accept vinyl records as a medium of live performance in the context of Rhythmic Hip Hop and Electronic Dance Music (EDM).

\section{C: Easy-Going Housework with Unpretentious and Rhythmic Music}

Housework is often felt as being a boring, monotonous and partly alienating duty by all of our young informants. To enrich their experience of this practice, members of the Versatile Audiophile cluster draw on music of the Unpretentious or Rhythmic style, playing it back from their HiFi stereo on a very high volume while working, such as Tim:

$R$ : Yes and regarding cleaning-up, it's all about doing something what you not exactly like doing, and adding a somehow more positive basic mood. I: Hmm, and how loud would you put on your music then?

R: Well, that depends. I have to admit, you somehow develop the feeling, the longer you listen the more tolerant you get to turn it louder every time.

As it turns out, listening at high volume seems to be central to the situated activity of cleaning up for all members of the cluster, even if the specific reasons often cannot be explicated: When being forced to temporary living at his parents flat again after finishing his study and being requested to clean up his room, Tim even sets up his old HiFi appliance again for this duty, thereby re-enacting a practice he got used to through the study years. While every informant from the Versatile Audiophiles depicted this type of embodied activity accompanied by loud music (sometimes without being really able to explain why), virtually nobody from the Digital Mobilists was mentioning it at all as own practice, even if their 
narrowed repertoire would in principle also allow them to listen to music with high volume via the headphones of their mobile players.

\section{D: Collective Gaming and Work-Out with Rhythmic and Intense Music}

A significant amount of our informants likes to play video and computer games. When being absorbed in the virtual worlds and playing together with friends, they typically rely on the auditory navigational information they receive from the game engine in the form of spatially localized, interactive sound effects. Conversely, when the game employs an additional music sound track, this is often felt as boring or distracting and therefore turned off and substituted by own music from mobile player, computer or smartphone that is eventually connected to a HiFi stereo, active speakers or a docking station. Noah narrates:

I: Do you know situations where you couldn't do without music?

$R$ : When I use my Playstation.

I: That so?

R: Yes, I am not gonna do without it. Recently, with a mate, we had been listening to music all the time, different songs, and then suddenly the music was off, because the last song had ended and then we played for a while and then we were like: 'something's missing somehow!' [laughs]. Yes, and that's why I couldn't do without it.

Even if the music is not specially tailored and synchronized to the gameplay, it is nevertheless felt by our informants to help with getting into the flow or feel of the gaming experience. Hence, Versatile Audiophile informants put on their stereo unit in parallel to the game console and listen to their preferred music, which is often from the Intense or Rhythmic style, depending on the type of game. While Rhythmic music seems to help to with getting into the flow of driving and navigating, intense music seems to be well-fitted to produce aggression and adrenaline needed for more competitive or combat-driven gaming activities. This logic finds its parallel in other collective activities of Versatile Audiophile members like jogging or work-out. For example, when working-out in the cellar together with his best friends, our informant Tim typically draws on special Intense music mixes that help them to 'press out the last rest of force' when in danger of losing their motivation. Both these types of very bodyrelated, highly-functionalist music employment accompanied by collective physical exercise or virtual gameplay is described by none of the informants we interviewed from the Digital Mobilists and seem also closely related to the experience of getting physiologically affected by moody vibration, pulse and volume of Intense or Rhythmic music.

\section{DISCUSSION}

The present paper tried to empirically synthesize existing research discourses on music socialization and musical taste in order to explain audio repertoire composition. Besides replicating prior findings on the influence of birth cohort and gender, an effect of audio socialization could be identified as possible further explanatory mechanism of audio repertoire genesis. Additionally, we found covariations of musical genre preferences with audio repertoire composition confirming our understanding that both might have similar music media orientations as a common cause. The inclusion of genre preferences in the model also helped to better understand the effects of gender on individual audio repertoire composition. Finally, our Grounded Theory analysis of narrative interviews with selected members of the two audio repertoire classes in focus resulted in identification of two 
contrasting modes of embodied musical experience that are situation-specifically adapted in the everyday. The description of their logics and retrodiction of their genesis substantially enriched interpretation of the correlations found in the survey study.

\subsection{Synthesis of Survey Study Results}

\subsubsection{Influence of Cohort and Gender on Audio Repertoire Class Membership}

In general, the frequencies of audio repertoire class members found in the student sample met what could be expected from prevalences within the overall population estimated by the representative pilot study, even when working with merely more than a third of the original questionnaire items. Also, dimensionality of musical taste preferences and their exogenous correlations with socio-demographics were in line with the state of research literature, as were the findings of the first regression model: For first-year students, birth cohort membership and gender significantly determine being either member of the Versatile Audiophiles or the Digital Mobilists.

\subsubsection{Influence of Music and Audio Socialization on Audio Repertoire Composition}

The results of the second model step gave rise to the additional interpretation that controlling for gender and birth cohort, being member of the Versatile Audiophiles is rather a product of intensified audio socialisation experience than of formal music socialization. This conclusion is underpinned by the fact that the latter variable was still able to explain large variance proportions in individual music taste preferences and therefore seems validly measured.

\subsubsection{Covariations between Musical Taste, Gender and Audio Repertoire}

When implementing the third model step, it became obvious that the observed gender effect on repertoire composition may partly be explained by apparently gender related musical taste preferences: Female students of the given student sample show a higher affinity to Mellow music and a lesser to Rhythmic and Unpretentious Styles and the males vice versa. At the same time, adherents of the latter styles tend to rather belong to the Versatile Audiophile repertoire, while Digital Mobilists are more affine to Mellow music. Due to the crosssectional character of the dataset and the socially selective sample, it is impossible to establish a temporal direction of causality between audio repertoire and musical taste patterns from this finding, so we would carefully interpret results as a demi-regular contiguity of musical taste preferences and habitual technology selection that seems to ground in partly gender-specific audio socialization experiences within the socio-technological milieux of childhood and adolescence.

\subsection{Complementary Results from the Interview Study}

\subsubsection{The Role of Media and Music Socialization}

When trying to reconstruct the specifics of the empirically identified audio socialization effect on repertoire development from the narrative material of interviewed adolescents and young adults, it became obvious that the (predominantly male) Versatile Audiophiles were 
often supported by their parents financially and ideologically to develop their amateur abilities. Sometimes also the mere breadth of available audio technologies in a family household due to a higher economic background seemed to contribute to developing a more 'versatile' usage pattern in later life, confirming the effect of income found in the populationrepresentative pilot study. The social capital and prestige gathered by acquiring, presenting and being able to handle diverse technologies seemed to play an additional self-reinforcing role in this regards, too.

\subsubsection{Explaining the Gender Differences}

When contrasting depictions of music listening from both audio repertoire classes in focus, we became aware of two contrasting dominant music media orientations that may be subsumed as functional vs. expressive mode and could be directly projected onto repertoire class membership and preferred music listening dispositifs. In their biographical genesis, the dominance of one of the two modes appears to be partly explainable by gender-specific audio enculturation practices within the peer-group encouraging the functional mode for males, since stereotypical depictions of respective male peer-group HiFi-enthusiasm and related symbolical exchanges were narrated by informants of either gender and either audio repertoire class.

\subsubsection{The Role of Musical Taste Preferences}

Even if also the interview study was not able to clarify the actual direction of causality between musical taste preferences and audio repertoire composition, it could shed further light on the found contiguities between a female affinity for the Mellow Music Genre and being a Digital Mobilist. It appears that they are both outcomes of a strong habitual symbolic orientation with regards to music listening practices that centres on soulful re-enactments of social memories by help of certain 'holy' music titles and the mobilization of the symbolic 'aura' of material devices and storage media in a calm and solipsistic atmosphere, while the contiguity of Rhythmic, Unpretentious musical preferences with being a Versatile Audiophile seems to be partly explainable by a functionalist passion for embodied experience with the arousing physiological effects of loud music in terms of bass vibrations, pulsating rhythm experience or aggressive mood in collective work out and game situations that require the use of high volume speakers and genre-specific playlists that may be transferred across audio media platforms. These findings may be interpreted as a media-related complement to the theory of 'body genres' put forward by Meier (2008).

\subsubsection{Overall Limitations of Interpretation}

A limitation of the present results is that the survey sample drew on participants from a comparatively higher income and educational background than a normal population. Therefore, educational and economic stratification of social mechanisms responsible for audio repertoire formation could not be put in focus by the regression model and the results and their interpretations may therefore exhibit a hidden educational and income bias. Accordingly and due to the small volume of only three informants per audio repertoire class, we also did not systematically address this issue in the interview study. Future empirical work should try to shed more light on these dimensions, since there are clear empirical findings that social- 
economic heterogeneities regarding audio media use and musical taste indeed exist in the German adolescent population (Gross and Walden 2014).

\subsection{Outlook: Towards New Forms of Media Dispositif Analysis}

While the results at hand helped us with further clarifying the research questions on relations between socialization, musical preferences and audio repertoires, they nevertheless also show the limits of current empirical social research methodology when it comes to analysing the complex communicative figurations of music listening in everyday life, even when employing a mixed-methods approach: Habitual media use always has to be retrospectively reconstructed, be it in surveys or in interview studies, thereby rendering the finding of homogenous patterns and dependencies a difficult endeavour. For further clarifying the complex relationships between media and music use that might also include latent spatial and temporal rhythmicity, it would therefore seem desirable to gain more real-time behavioural data on habitual situated media use combined with interview studies or think-aloud techniques that help with complementarily understanding the observed practices. Future studies should consider drawing on smartphone-based Experience Sampling Methodology (Kubey, Larson and Csikszentmihalyi 1996) to achieve these aims.

\section{References}

Baacke, D., Sander, U. and Vollbrecht, R. (1990) Lebenswelten sind Medienwelten, Opladen: Leske Budrich.

Collins, L. M. and Lanza, S. T. (2010) Latent Class and Latent Transition Analysis. With Applications in the Social, Behavioral, and Health Sciences, Hoboken (NJ), USA: Wiley.

DeNora, T. (1999) 'Music as a technology of the self', Poetics, 27(1), 31-56.

Dollase, R. (1998) 'Musikpräferenzen und Musikgeschmack Jugendlicher', in D. Baacke (ed.), Handbuch Jugend und Musik, Opladen: Leske \& Budrich, pp. 341368.

Gebesmair, A. (2001) Grundzüge einer Soziologie des Musikgeschmacks, Opladen: Westdeutscher Verlag.

Gross, F. von, \& Walden, T. (2013) 'Jugendlicher Bildungshintergrund und Musikpräferenz', in R. Heyer, S. Wachs, and C. Palentien (eds), Handbuch Jugend Musik - Sozialisation, Wiesbaden: Springer Fachmedien, pp. 343-370.

Hallam, S. (2002) 'Musical Motivation: Towards a model synthesising the research', Music Education Research, 4(2), 225-244.

Hepp, A. (2013) 'The Communicative Figurations of Mediatized Worlds: Mediatization Research in Times of the "Mediation of Everything"', European Journal of Communication, 28(6), 615-629. 
Jost, E. (1982) 'Sozialpsychologische Dimensionen des Musikalischen Geschmacks', in C. Dahlhaus and H. de la Motte-Haber (eds), Systematische Musikwissenschaft, Wiesbaden: Anthenaion, pp. 245-268.

Keightley, K. (2003) 'Low Television, High Fidelity: Taste and the Gendering of Home Entertainment Technologies', Journal of Broadcasting \& Electronic Media, 47(2), 236-259.

Krämer, B. (2013) Mediensozialisation: Theorie und Empirie zum Erwerb medienbezogener Dispositionen, Wiesbaden: Springer VS.

Krotz, F. (2014) 'Mediatization as a Mover in Modernity: Social and Cultural Change in the Context of Media Change', in K. Lundby (ed.), Mediatization of Communication, Berlin/Boston: de Gruyter, pp. 131-161.

Kubey, R. W., Larson, R. and Csikszentmihalyi, M. (1996) 'Experience Sampling Method Applications to Communication Research Questions', Journal of Communication, 46(2), 99-120.

Kunz, A. (1998) Aspekte der Entwicklung des persönlichen Musikgeschmacks, Frankfurt am Main: Lang.

Leguina, A., Arancibia-Carvajal, S., \& Widdop, P. (2015) 'Musical Preferences and Technologies: Contemporary Material and Symbolic Distinctions Criticized', Journal of Consumer Culture, online first article.

Lenz, F. (2013) 'Soziologische Perspektiven auf musikalische Sozialisation', in R. Heyer, S. Wachs and C. Palentien (eds), Handbuch Jugend - Musik - Sozialisation, Springer Fachmedien Wiesbaden, pp. 157-185.

Lepa, S., Hoklas, A.-K. and Weinzierl, S. (2014) 'Discovering and Interpreting Audio Media Generation Units: A Typological-Praxeological Approach to the Mediatization of Everyday Music Listening', Participations, 11(2), 207-238.

Lepa, S., Krotz, F. and Hoklas, A.-K. (2014) 'Vom ,Medium' zum ,Mediendispositiv': Metatheoretische Überlegungen zur Integration von Situations- und Diskursperspektive bei der empirischen Analyse mediatisierter sozialer Welten', in F. Krotz, C. Despotovic and M. Kruse (eds), Die Mediatisierung sozialer Welten: Synergien empirischer Forschung, Wiesbaden: VS - Verlag für Sozialwissenschaften, pp. $115-141$.

Lepa, S. and Seifert, M. (in press) 'Musikalische Vorlieben oder Alltagsästhetische Schemata? Zur relativen Bedeutung von Demographie-, Sozialisations- und Persönlichkeitsvariablen für die Optimierung digitaler Musikempfehlungssysteme', Jahrbuch der Deutschen Gesellschaft für Musikpsychologie, 24.

Mannheim, K. (1952) 'The Sociological Problem of Generations', in Essays on the Sociology of Knowledge, Oxford: Oxford University Press, pp. 163-195.

Marsh, H. W., Muthén, B., Asparouhov, T., Lüdtke, O., Robitzsch, A., Morin, A. J. S. and Trautwein, U. (2009) 'Exploratory Structural Equation Modeling, Integrating CFA and EFA: Application to Students' Evaluations of University Teaching', Structural Equation Modeling: A Multidisciplinary Journal, 16(3), 439-476. 
McPherson, G. E. (2009) 'The Role of Parents in Children's Musical Development', Psychology of Music, 37(1), 91-110.

Meier, L. M. (2008) 'In Excess? Body Genres, "Bad" Music, and the Judgment of Audiences', Journal of Popular Music Studies, 20(3), 240-260.

Miranda, D., Blais-Rochette, C., Vaugon, K., Osman, M. and Arias-Valenzuela, M. (2015) 'Towards a Cultural-Developmental Psychology of Music in Adolescence', Psychology of Music, 43(2), 197-218.

Müller-Bachmann, E. (2002) Jugendkulturen Revisited: Musik- und Stilbezogene Vergemeinschaftungsformen (Post-)Adoleszenter im Modernisierungskontext, Münster: LIT Verlag.

Muthén, L. K. and Muthén, B. O. (2010) Mplus User's Guide. Statistical Analysis with Latent Variables. Sixth Edition, Los Angeles, CA (USA): Muthén \& Muthén.

Quiñones, M. G. (2013) 'Body and Context in Mobile Listening to Digital Players', in M. G. Quiñones, A. Kassabian and E. Boschi (eds), Ubiquitous Musics: The Everyday Sounds That We Don't Always Notice, Farnham: Ashgate, pp. 107-118.

Peterson, R. A., and Kern, R. M. (1996) 'Changing Highbrow Taste: From Snob to Omnivore', American Sociological Review, 61(5), 900-907.

Reinhardt, J. and Rötter, G. (2013) 'Musikpsychologischer Zugang zur JugendMusik-Sozialisation', in R. Heyer, S. Wachs and C. Palentien (eds), Handbuch Jugend - Musik - Sozialisation, Wiesbaden: Springer, pp. 127-155.

Rentfrow, P. J., Goldberg, L. R. and Levitin, D. J. (2011) 'The Structure of Musical Preferences: A Five-Factor Model', Journal of Personality and Social Psychology, 100(6), 1139-1157.

Schäfer, T. and Sedlmeier, P. (2010) 'What Makes us Like Music? Determinants of Music Preference', Psychology of Aesthetics, Creativity, and the Arts, 4(4), 223-234.

Schramm, H. (2005) Mood Management durch Musik. Die alltägliche Nutzung von Musik zur Regulierung von Stimmungen, Köln: Herbert von Halem.

Schulze, G. (1992) Die Erlebnis-Gesellschaft. Kultursoziologie der Gegenwart, Frankfurt am Main: Campus.

Taubert, P. (2006) Lebensstile und Mediennutzung. Theoretische Grundlagen und empirische Umsetzung, München: Martin Meidenbauer. 
Steffen Lepa is postdoc researcher at Audio Communication Group, Technische Universität Berlin, Germany, principal investigator of the research project 'Music and Media Survey. Empirical Basic Data and Theoretical Modeling of the Mediatization of Everyday Music Reception in Germany' and affiliated with Berlin cluster-of-excellence research project 'Medium and emotion. On the Emotional Effects of Music under Various Media Conditions'. Research interests: Mediatization Research, Audio Reception, Media Use, Methodology of Social Sciences, Media Education, Sound Design, Popular Culture.

Email: steffen.lepa@tu-berlin.de

Markus Seifert is postdoc researcher at the Department for Media and Communication Studies, Universität Erfurt, Germany and principal investigator of the research project 'Mooddependent Reception of Music'. Research interests: Media and Music Reception of Children, and Adolecents, Media Socialization and Musical Taste Preferences.

Email: markus.seifert@uni-erfurt.de 